\title{
STRUCTURE OF STABLE MINIMAL HYPERSURFACES IN A RIEMANNIAN MANIFOLD OF NONNEGATIVE RICCI CURVATURE
}

\author{
JEONG-Jin Kim AND GABJIN Yun
}

\begin{abstract}
Let $N$ be a complete Riemannian manifold with nonnegative Ricci curvature and let $M$ be a complete noncompact oriented stable minimal hypersurface in $N$. We prove that if $M$ has at least two ends and $\int_{M}|A|^{2} d v=\infty$, then $M$ admits a nonconstant harmonic function with finite Dirichlet integral, where $A$ is the second fundamental form of $M$. We also show that the space of $L^{2}$ harmonic 1 -forms on such a stable minimal hypersurface is not trivial. Our result is a generalization of one of main results in [12] because if $N$ has nonnegative sectional curvature, then $M$ admits no nonconstant harmonic functions with finite Dirichlet integral. And our result recovers a main theorem in [3] as a corollary.
\end{abstract}

\section{Introduction}

The classical Bernstein theorem asserts that an entire minimal graph in $\mathbb{R}^{3}$ must be planar. This theorem was subsequently generalized to higher dimensions by several authors, cf. [1], [5], [9], [17]. It is now known [2], [17] that an entire $n$-dimensional minimal graph in $\mathbb{R}^{n+1}$ must be given by a linear function over $\mathbb{R}^{n}$ providing that $n \leq 7$ and for $n \geq 8$ there are nonlinear entire minimal graphs in $\mathbb{R}^{n+1}$. Since minimal graphs are area minimizing, one can consider a generalization of the Bernstein problem to a larger class of submanifolds given by the stable minimal hypersurfaces in $\mathbb{R}^{n+1}$.

DoCarmo and Peng [6] proved that a complete stable minimally immersed hypersurface in $\mathbb{R}^{3}$ must be planar. And, Fischer-Colbrie and Schoen [8] showed independently that a complete stable minimally immersed hypersurface $M$ in a complete 3-dimensional manifold $N$ with nonnegative scalar curvature must

Received August 3, 2012.

2010 Mathematics Subject Classification. 53C21.

Key words and phrases. stable minimal hypersurface, end, $L^{2}$ harmonic form, parabolicity, non-parabolicity.

The second author was supported by the Basic Science Research Program through the National Research Foundation of Korea(NRF) funded by the Ministry of Education, Science and Technology(Grant No. 2012-0002635). 
be either conformally a plane $\mathbb{R}^{2}$ or conformally a cylinder $S^{1} \times \mathbb{R}$. For the special case when $N$ is $\mathbb{R}^{3}$, they also proved that $M$ must be planar.

Furthermore, using curvature estimates of minimal hypersurfaces described as in [14], DoCarmo and Peng [7] showed that if $M$ is an oriented stable complete minimal hypersurface in $\mathbb{R}^{n+1}$ such that $\int_{M}|A|^{2} d v_{g}<\infty$, then $M$ is a hyperplane. In case of symmetric spaces, the second author [19] proved that $M$ is an oriented stable complete minimal hypersurface in a locally symmetric space of nonnegative Ricci curvature such that $\int_{M}|A|^{2} d v_{g}<\infty$, then $M$ must be totally geodesic.

On the other hand, Cao, Shen and Zhu [3] proved a topological property for stable minimal hypersurfaces in Euclidean space. Namely, they proved that a complete oriented stable minimal hypersurface $M^{n}$ in $\mathbb{R}^{n+1}$ must have only one end. In [12], Li and Wang generalized this result. They showed that if $M$ is a complete stable minimal hypersurface in a complete manifolds $N$ with nonnegative sectional curvature, then either $M$ has only one end, or $M$ splits into $\Sigma \times \mathbb{R}$ with product metric, and in the second case $M$ is totally geodesic in $N$. We generalize this result to the case that $N$ has nonnegative Ricci curvature. Our approach is to use the theory of harmonic functions with finite energy and $L^{2}$ harmonic differential forms together with non-parabolicity of Riemannian manifolds. The existence or non-existence of $L^{2}$ harmonic differential forms are related to the structure of oriented minimal hypersurfaces in a Riemannian manifold. For instance, Miyaoka proved [13] that if $M$ is an oriented stable minimal hypersurface in a Riemannian manifold $N$ with nonnegative sectional curvature, then there are no non-trivial $L^{2}$ harmonic one forms on $M$. In case of minimal hypersurfaces, it is well known [15], [20] that if the total scalar curvature of $M$ is sufficiently small, then there are no non-trivial $L^{2}$ harmonic one forms on $M$. On the other hand, parabolicity or non-parabolicity of a Riemannian manifolds is deeply related with the number of ends of a given Riemannian manifold, cf. [11].

\section{Parabolicity and stability}

From now on, we assume that $N^{n+1}$ is a complete Riemannian manifold of dimension $n+1$ otherwise stated. We say that a minimally immersed hypersurface $M$ into $N$ is stable if the second derivative of the area functional is nonnegative for any compactly supported normal variation. This is equivalent [4] that for any function $\varphi \in C_{0}^{1}(M)$,

$$
\int_{M}|\nabla \varphi|^{2}-\left(|A|^{2}+\overline{\operatorname{Ric}}(\nu, \nu)\right) \varphi^{2} \geq 0
$$

where $A$ denotes the second fundamental form of $M, \nu$ is an unit normal vector field on $M$, and $\overline{\mathrm{Ric}}$ is the Ricci curvature of $N$.

We start with a well-known result due to Fischer-Colbrie and Schoen and an easy observation from it. 
Lemma 2.1 ([8]). Let $M$ be a complete noncompact stable minimal hypersurface in $N$. Then there exists a globally defined positive function $u>0$ on $M$ satisfying

$$
\Delta u+\left(|A|^{2}+\overline{\operatorname{Ric}}(\nu, \nu)\right) u=0 .
$$

We say that a Riemannian manifold $M$ is non-parabolic if it admits a positive Green function. Otherwise we say that it is parabolic. It is well-known that a Riemannian manifold $M$ is non-parabolic if and only if it admits a non-constant positive superharmonic function on it.

It is also well-known [10], [18] that a non-parabolic Riemannian manifold satisfies

$$
\int_{1}^{\infty} \frac{t}{\operatorname{vol}\left(B_{p}(t)\right)} d t<\infty
$$

where $B_{p}(t)$ is a geodesic ball in $M$ of radius $t$ centered at a point $p$. In particular, a non-parabolic manifold has an infinite volume. For parabolicity or Green functions, one can refer [11] and references are therein.

An easy observation about parabolicity for minimal hypersurfaces is the following.

Lemma 2.2. Let $M$ be a complete noncompact stable minimal hypersurface in a complete Riemannian manifold $N$ with $\overline{\mathrm{Ric}} \geq 0$. Then $M$ is non-parabolic or totally geodesic.

Proof. Suppose $M$ is parabolic. It follows from Lemma 2.1 that there exists a positive function $u$ satisfying

$$
\Delta u+\left(|A|^{2}+\overline{\operatorname{Ric}}(\nu, \nu)\right) u=0 .
$$

In particular, $u$ is superharmonic since $\overline{\operatorname{Ric}} \geq 0$. Since $M$ is parabolic, $u$ must be constant and so

$$
\left(|A|^{2}+\overline{\operatorname{Ric}}(\nu, \nu)\right) u=0 .
$$

This implies $A=0$ and $\overline{\operatorname{Ric}}(\nu, \nu)=0$.

\section{Ricci curvature and structure of ends}

For a complete oriented stable minimal submanifold $M$ in a complete Riemannian manifold $N$, the integral of the square norm of the second fundamental form $A$ on $M, \int_{M}|A|^{2} d v$, plays an important role in the structure of $M$. For instance, if $M$ is an oriented, complete, stable minimal hypersurface in $\mathbb{R}^{n+1}$ such that $\int_{M}|A|^{2} d v<\infty$, then $M$ is a totally geodesic and so is a hyperplane [7], [14]. Here $d v$ denotes the volume form of the induced metric on $M$ and we will abbreviate it from now if there is no ambiguity.

The following theorem shows that if $M$ is a complete stable minimally immersed hypersurface in a complete Riemannian manifold $N$ with nonnegative Ricci curvature and if $\int_{M}|A|^{2} d v=\infty$, then $M$ has only one end, or $M$ admits a nonconstant harmonic function with finite energy. Using the similar way as 
in [3] if replacing a Sobolev inequality by the stability condition, we can prove this result.

Theorem 3.1. Let $M^{n}$ be a noncompact complete oriented stable minimal hypersurface in $N^{n+1}$ with nonnegative Ricci curvature. If $M$ has at least two ends and satisfies $\int_{M}|A|^{2}=\infty$, then $M$ admits a non-constant harmonic function with finite Dirichlet integral.

Proof. Suppose $M$ has at least two ends and $\int_{M}|A|^{2}=\infty$. Let $\left\{D_{i}\right\}$ be an exhaustion of $M$ of smooth boundary and let

$$
M-D_{i}=\sum_{j=1}^{m} E_{j}^{(i)} .
$$

Let $u_{i}$ be the solution of

$$
\Delta u_{i}=0 \quad \text { on } D_{i}
$$

and the boundary conditions

$$
\left.u_{i}\right|_{\partial E_{1}^{(i)}}=1,\left.\quad u\right|_{\partial E_{k}^{(i)}}=0 \quad(k \geq 2) .
$$

By maximum principle, $0 \leq u_{i} \leq 1$ and

$$
\int_{D_{i}}\left|\nabla u_{i}\right|^{2} \leq \int_{D_{j}}\left|\nabla u_{j}\right|^{2} \quad(j \leq i) .
$$

Thus $u_{i}$ converges to a harmonic function $u$ satisfying

$$
0 \leq u \leq 1, \quad \int_{M}|\nabla u|^{2} \leq C_{1}
$$

for some constant $C_{1}>0$. We claim that $u$ is not constant. Note that

$$
\begin{aligned}
\int_{D_{i}}\left(|A|^{2}+\overline{\operatorname{Ric}}(\nu, \nu)\right)\left(u_{i}\left(1-u_{i}\right)\right)^{2} & \leq \int_{D_{i}}\left|\nabla\left(u_{i}\left(1-u_{i}\right)\right)\right|^{2} \\
& \leq 9 \int_{D_{i}}\left|\nabla u_{i}\right|^{2} \leq 9 C_{1} .
\end{aligned}
$$

Thus if $u$ is constant, then by (3.2), $u=0$ or $u=1$ since $\int_{M}|A|^{2}=\infty$ and $\overline{\operatorname{Ric}}(\nu, \nu) \geq 0$. We may assume $u=1$ by replacing $u$ by $1-u$ if necessary.

Now for a fixed $i_{0}$, since $\int_{M}|A|^{2}=\infty$, there exists an end, say $E_{l}^{\left(i_{0}\right)}$, such that

$$
\int_{E_{l}^{\left(i_{0}\right)}}|A|^{2}=\infty .
$$

We may assume $l \neq 1$ by changing the boundary conditions in (3.1) if necessary. Choose a smooth function $\psi$, for the fixed $i_{0}$, satisfying

$$
\psi=\left\{\begin{array}{lll}
1 & \text { on } & E_{l}^{\left(i_{0}\right)} \\
0 & \text { on } & E_{k}^{\left(i_{0}\right)}
\end{array} \quad(k \neq l)\right.
$$


and

$$
|\nabla \psi| \leq C_{2}, \quad 0 \leq \psi \leq 1
$$

for some constant $C_{2}>0$ independent of $i$ and $u_{i}$. Note that $|\nabla \psi|$ vanishes outside a compact set. Moreover, since $\left.u_{i}\right|_{\partial E_{1}^{(i)}}=1$ and $\left.u_{i}\right|_{\partial E_{k}^{(i)}}=1$ for $k \geq 2$, and $l \neq 1$, the function $\phi_{i}=u_{i} \psi$ vanishes on $\partial D_{i}$. Thus the stability condition (2.1) shows that

$$
\begin{aligned}
\int_{D_{i}}\left(|A|^{2}+\overline{\operatorname{Ric}}(\nu, \nu)\right) \phi_{i}^{2} & \leq \int_{D_{i}}\left|\nabla \phi_{i}\right|^{2} \\
& \leq 2 \int_{D_{i}}\left(\left|\nabla u_{i}\right|^{2}+|\nabla \psi|^{2}\right) \leq C_{3},
\end{aligned}
$$

where $C_{3}$ is a positive constant independent of $i$ and $u_{i}$. Thus

$$
\int_{E_{l}^{\left(i_{0}\right)} \cap D_{i}}\left(|A|^{2}+\overline{\operatorname{Ric}}(\nu, \nu)\right) u_{i}^{2} \leq C
$$

and by letting $i \rightarrow \infty$, we obtain

$$
\int_{E_{l}^{\left(i_{0}\right)}}|A|^{2} \leq \widetilde{C}
$$

which contradicts (3.3). Consequently, the limit function $u$ of $u_{i}$ is a nonconstant harmonic function with finite Dirichlet integral.

Remark 3.2. The condition $\int_{M}|A|^{2}=\infty$ is necessary. For instance, let $M=$ $S^{n-1} \times \mathbb{R} \subset S^{n-1} \times \mathbb{R}^{2}$ so that $M$ is a totally geodesic hypersurface in $S^{n-1} \times \mathbb{R}^{2}$. It is well-known that $M=S^{n-1} \times \mathbb{R}$ has no nonconstant harmonic function with finite Dirichlet integral. It follows from this fact together with Lemma 2.2 that if $M=S^{n-1} \times \mathbb{R}$ is a stable minimal hypersurface in any Riemannian manifold $N$ of nonnegative Ricci curvature, then $M$ must be totally geodesic.

Note that the total differential of a nonconstant harmonic function defined on a noncompact complete Riemannian manifold is a nontrivial harmonic oneform. A differential form $\omega$ on a Riemannian manifold $M$ is called $L^{2}$ harmonic if it satisfies

$$
\Delta \omega=(d \delta+\delta d) \omega=0, \text { and } \int_{M} \omega \wedge * \omega=\int_{M}|\omega|^{2} d v<\infty,
$$

where $*$ denotes the Hodge star operator and $d v$ is the volume form on $M$. The space of harmonic differential forms on a noncompact Riemannian manifold does not satisfy the Poincaré duality in general. But the space of $L^{2}$ harmonic forms dose satisfy the Poincaré duality. That is, the space of $L^{2}$ harmonic $p$ forms is isomorphic to the space of $L^{2}$ harmonic $(n-p)$-forms, where $\operatorname{dim}(M)=$ $n$.

Note that if $u$ is a nonconstant harmonic function with finite Dirichlet integral, then the total differential, $d u$, is a nontrivial $L^{2}$ harmonic one-form on $M$. Thus non-existence of $L^{2}$ harmonic one forms implies the non-existence of 
non-constant harmonic functions with finite Dirichlet integral. From this fact together with Theorem 3.1, we have the following theorem.

Theorem 3.3. Let $M^{n}$ be a noncompact complete oriented stable minimal hypersurface in $N^{n+1}$ with nonnegative Ricci curvature. If $M$ has at least two ends and $\int_{M}|A|^{2}=\infty$, then both the space of $L^{2}$ harmonic one-forms and the space of $L^{2}$ harmonic $(n-1)$-forms are non-trivial.

As mentioned above, if $M$ is a complete oriented stable minimal hypersurface into a complete Riemannian manifold $N$ and the sectional curvature of $N, \bar{K}_{N}$, is nonnegative, then $M$ does not admit nontrivial $L^{2}$ harmonic one-forms.

Theorem 3.4 ([13]). Let $M$ be a complete, oriented, stable minimal hypersurface into a complete Riemannian manifold $N$. If the sectional curvature of $N$ is nonnegative, then $M$ does not admit nontrivial $L^{2}$ harmonic one-forms.

Theorem 3.1 together with Theorem 3.4 implies the following corollary which is a main result in [3]. We would like to mention that in [3], they assumed $n \geq 3$ because of the exponent of the Sobolev inequality. However our proof for Theorem 3.1 or Theorem 3.3 does not use the Sobolev inequality and so we can extend their result to $n \geq 2$. We would remark that when $n=2$, DoCarmo and Peng [6], and Fischer-Colbrie and Schoen [8] proved independently that a complete stable minimal surface in $\mathbb{R}^{3}$ is a plane.

Corollary 3.5. If $M$ is a complete stable minimal hypersurface in $\mathbb{R}^{n+1}$ with $n \geq 2$, then $M$ must have only one end.

Proof. If $M$ is a complete stable minimal hypersurface in $\mathbb{R}^{n+1}$, then $M$ has only one end or $\int_{M}|A|^{2}<\infty$ by Theorem 3.1. If $\int_{M}|A|^{2}<\infty$, then $M$ must be planar by [7] and so $M$ has only one end in this case, too.

Finally, in [16], Shen and Zhu studied the topology or rigidity of a stable minimal hypersurface of the product type $\Sigma \times \mathbb{R}$ in a complete Riemannian manifold $N$, where $\Sigma$ is a compact smooth manifold. Related to this result, we obtain the following result from our main result.

Corollary 3.6. Let $\Sigma$ be a compact smooth manifold and let $M=\Sigma^{n-1} \times \mathbb{R}$ be a stable minimal hypersurface in a complete Riemannian manifold $N^{n+1}$ with nonnegative Ricci curvature. If $\int_{M}|A|^{2}=\infty$, then the spaces of both $L^{2}$ harmonic 1-forms and $L^{2}$ harmonic $(n-1)$-forms on $M$ are non-trivial.

\section{References}

[1] F. Almgren, Jr., Some interior regularity theorems for minimal surfaces and an extension of Bernstein's Theorem, Ann. of Math. (2) 84 (1966), 277-292.

[2] E. Bombieri, E. DeGiorgi, and E. Guisti, Minimal cones and the Bernstein problem, Invent. Math. 7 (1969), 243-268.

[3] H.-D. Cao, Y. Shen, and S. Zhu, The structure of stable minimal hypersurfaces in $\mathbf{R}^{n+1}$, Math. Res. Lett. 4 (1997), no. 5, 637-644. 
[4] S. S. Chern, Minimal Submanifolds in a Riemannian Manifolds, University of Kansas, 1968.

[5] E. DeGiorgi, Una estensione del teorema di Bernstein, Ann. Scuola Norm. Sup. Pisa 19 (1965), 79-85.

[6] M. do Carmo and C. K. Peng, Stable complete minimal surfaces in $\mathbb{R}^{3}$ are planes, Bull. Amer. Math. Soc. (N.S.) 1 (1979), no. 6, 903-906.

[7] _ Stable minimal hypersufaces, Proceedings of the 1980 Beijing Symposium on Differential Geometry and Differential Equations, Vol. 1, 2, 3 (Beijing, 1980), 1349-1358, Sci. Press Beijing, Beijing, 1982.

[8] D. Fischer-Colbrie and R. Schoen, The structure of complete stable minimal surfaces in 3-manifolds of nonnegative scalar curvature, Comm. Pure Appl. Math. 33 (1980), no. $2,199-211$.

[9] W. Fleming, On the oriented Plateau problem, Rend. Circ. Mat. Palerimo 11 (1962), 69-90.

[10] A. Grigor'yon, On the existence of positive fundamental solution of the Laplacian equation on Riemannian manifolds, Math. USSR Sbornik 56 (1987), 349-358.

[11] P. Li, Curvature and Function Theory on Riemannian Manifolds, Surveys in differential geometry, 375-32, Surv. Differ. Geom., VII, Int. Press, Somerville, MA, 2000.

[12] P. Li and J. Wang, Stable minimal hypersurfaces in a nonnegatively curved manifold, J. Reine Angew. Math. 566 (2004), 215-230.

[13] R. Miyaoka, Harmonic 1-forms on a complete stable minimal hypersurfaces, Geometry and global analysis (Sendai, 1993), 289-293, Tohoku Univ., Sendai, 1993.

[14] R. Schoen, L. Simon, and S.-T. Yau, Curvature estimates for minimal hypersurfaces, Acta Math. 134 (1975), no. 3-4, 275-288.

[15] K. Seo, Rigidity of minimal submanifolds in hyperbolic space, Arch. Math. 94 (2010), no. 2, 173-181.

[16] Y. Shen and S. Zhu, Rigidity of stable minimal hypersurfaces, Math. Ann. 309 (1997), no. 1, 107-116.

[17] J. Simons, Minimal varieties in Riemannian manifolds, Ann. of Math. (2) 88 (1968), 62-05.

[18] N. Th. Varopoulos, Potential theory and diffusion on Riemannian manifolds, Conference on harmonic analysis in honor of Antoni Zygmund, Vol. I, II (Chicago, Ill., 1981), 821837, Wadsworth Math. Ser., Wadsworth, Belmont, CA, 1983.

[19] G. Yun, Stable minimal hypersurfaces in locally symmetric spaces, Math. Nachr. 280 (2007), no. 15, 1744-1751.

[20] - Total scalar curvature and $L^{2}$ harmonic 1-forms on a minimal hypersurface in Euclidean space, Geom. Dedicata 89 (2002), 135-141.

JEONG-JIN KIM

Department of Mathematics

MYONG Ji UNIVERSITY

YONGIN 449-728, KorEA

E-mail address: jjkim@mju.ac.kr

GABJIN YUN

Department of Mathematics

MyONG Ji UNIVERSITY

YONGIN 449-728, KorEA

E-mail address: gabjin@mju.ac.kr 